\title{
A three-dimensional acoustic Boundary Element Method formulation with viscous and thermal losses based on shape function derivatives
}

\section{Cutanda Henriquez, Vicente; Andersen, Peter Risby}

Published in:

Journal of Computational Acoustics

Link to article, DOI:

$10.1142 /$ S2591728518500391

Publication date:

2018

Document Version

Peer reviewed version

Link back to DTU Orbit

Citation (APA):

Cutanda Henriquez, V., \& Andersen, P. R. (2018). A three-dimensional acoustic Boundary Element Method formulation with viscous and thermal losses based on shape function derivatives. Journal of Computational Acoustics, 26(3), [1850039 ]. https://doi.org/10.1142/S2591728518500391

\section{General rights}

Copyright and moral rights for the publications made accessible in the public portal are retained by the authors and/or other copyright owners and it is a condition of accessing publications that users recognise and abide by the legal requirements associated with these rights.

- Users may download and print one copy of any publication from the public portal for the purpose of private study or research.

- You may not further distribute the material or use it for any profit-making activity or commercial gain

- You may freely distribute the URL identifying the publication in the public portal 


\title{
A three-dimensional acoustic Boundary Element Method formulation with viscous and thermal losses based on shape function derivatives
}

\author{
V. Cutanda Henríquez*, P. Risby Andersen ${ }^{\dagger}$ \\ Centre for Acoustic-Mechanical Micro Systems, Technical University of Denmark \\ Ørsteds Plads, Building 352, DK-2800, Kgs. Lyngby, Denmark \\ *vcuhe@elektro.dtu.dk \\ ${ }^{\dagger}$ prand@elektro.dtu.dk
}

Received (Day Month Year)

Revised (Day Month Year)

\begin{abstract}
Sound waves in fluids are subject to viscous and thermal losses, which are particularly relevant in the so-called viscous and thermal boundary layers at the boundaries, with thicknesses in the micrometer range at audible frequencies. Small devices such as acoustic transducers or hearing aids must then be modeled with numerical methods that include losses. In recent years, versions of both the Finite Element Method (FEM) and the Boundary Element Method (BEM) including viscous and thermal losses have been developed. This paper deals with an improved formulation in three dimensions of the BEM with losses which avoids the calculation of tangential derivatives on the surface by finite differences used in a previous BEM implementation. Instead, the tangential derivatives are obtained from the element shape functions. The improved implementation is demonstrated using an oscillating sphere, where an analytical solution exists, and a condenser microphone as test cases.
\end{abstract}

Keywords: boundary element method; viscous and thermal acoustic losses

\section{Introduction}

The effect of viscous and thermal losses in sound waves has been described long ago and is part of the general theory of physical acoustics. ${ }^{1,2,3}$. In free-field propagation in the audio range, such effects are only important over long distances such as in large auditoria, or outdoor sound propagation. However, they become very relevant at very close distance to the domain boundaries because: i) air molecules cannot slide over the boundary so that particle velocity must match boundary velocity in the normal and tangential directions, and ii) boundaries usually have such higher thermal conductivity than the fluid, that temperature variation is negligible at the boundary. The viscous and thermal boundary layers are generated by these effects, and are usually accounted for as boundary acoustic impedance. In the case of small setups with dimensions comparable to the boundary layer thicknesses, which vary from a fraction of a millimeter to a few micrometers, such approximation is not possible, and some other analytical or numerical model is necessary. Devices of this kind are acoustic transducers, couplers, hearing aids and small-scale acoustic metamaterials. ${ }^{4,5,6}$

For intricate and highly coupled devices, approximate solutions taking limiting hypothesis may not be sufficient for appropriate modeling. They must be tested or even replaced by full numerical models of the sound field with viscous and thermal losses. On the one 
hand, the Finite Element Method (FEM) can be employed for solving the no-flow linearized Navier-Stokes equations with no further hypotheses. ${ }^{7,8,9,10}$ The FEM implementation with losses is included in some commercial FEM software packages. ${ }^{11}$

On the other hand, an equally full implementation with losses uses the Boundary Element Method (BEM). ${ }^{12,13}$ The BEM is applied to the so-called viscous, thermal and acoustic modes resulting from the Kirchhoff decomposition of the Navier-Stokes equations. ${ }^{3,2,14}$ These three modes are coupled at the boundary with extended boundary conditions. The particle velocity boundary condition in the existing BEM with losses includes first and second tangential derivatives of the pressure at the boundary, which use finite differences and, as will be shown in this paper, can trigger numerical errors. The tangential derivative scheme is particularly cumbersome in the three-dimensional BEM implementation with losses. ${ }^{13}$

It is therefore desirable that the BEM with losses can be reformulated in a way that avoids finite difference intermediate calculations. One possibility is based on setting the tangential derivative as an initial variable to be solved for and reformulating the integral equations. ${ }^{15}$. In this paper, another alternative is presented, which preserves the integral equations but replaces the finite differences by derivatives of the element shape functions. The modified implementation is developed for three-dimensional domains and two examples are used to test its performance.

After this introduction, section two outlines the theoretical foundation on which the existing BEM for acoustics with viscous and thermal losses is based. Section three explains the new method for imposing the particle velocity boundary conditions. As a verification, section four presents an oscillating sphere test case where the new formulation is compared with the previous three-dimensional BEM implementation with losses and an analytical solution. Section five examines the performance of the new and existing formulations with a model of a condenser microphone. A discussion section comments the results of the tests, followed by a final section with conclusions and future work.

\section{Boundary Element Method formulation with losses}

The previously published three-dimensional BEM implementation with losses is described in detail in Ref. [13]. In this section, a short account of it is given with special focus on the issues that motivate this paper.

$$
\begin{gathered}
\left(\Delta+k_{a}^{2}\right) p_{a}=0 \\
\left(\Delta+k_{h}^{2}\right) p_{h}=0 \\
\left(\Delta+k_{v}^{2}\right) \vec{v}_{v}=\overrightarrow{0}, \text { with } \nabla \cdot \vec{v}_{v}=0
\end{gathered}
$$

Eqs. $(1,2,3)$ represent the three modes of the Kirchhoff decomposition of the NavierStokes equations on which the BEM implementation is based. ${ }^{2,14}$ Harmonic time dependence $e^{i \omega t}$ is omitted. The indexes $(a, h, v)$ indicate the so-called acoustic, thermal and viscous modes respectively, which can be treated independently in the acoustic domain and linked 
through the boundary conditions. The total pressure can be obtained as the sum $p=p_{a}+p_{h}$ of the acoustic and thermal components (there is not a viscous $p_{v}$ ), while the particle velocity has contributions from the three modes as $\vec{v}=\vec{v}_{a}+\vec{v}_{h}+\vec{v}_{v}$. The three wavenumbers $k_{a}$, $k_{h}$ and $k_{v}$ are function of the lossless wavenumber $k$ and the physical properties of the fluid: viscosity, bulk viscosity and thermal conductivity coefficients, air density, and specific heats. ${ }^{14}$ Eq.(1) is a wave equation, while Eqs. $(2,3)$ are diffusion equations. There are five equations and five unknowns because the viscous velocity $\vec{v}_{v}$ is a three-component vector and therefore Eq. (3) is split into three components.

$$
\begin{gathered}
T=T_{a}+T_{h}=\tau_{a} p_{a}+\tau_{h} p_{h}=0 \\
\vec{v}_{\text {boundary }}=\vec{v}_{a}+\vec{v}_{h}+\vec{v}_{v}=\phi_{a} \nabla p_{a}+\phi_{h} \nabla p_{h}+\vec{v}_{v}
\end{gathered}
$$

Eq. (4) states that the temperature $T$, the sum of its acoustic and thermal components $T_{a}$ and $T_{h}$, remains constant at the boundary, leading to a condition that links the thermal and acoustic pressures $p_{a}$ and $p_{h}$. Eq. (5) ensures that the total particle velocity, expressed as the sum of acoustic, thermal and viscous contributions, matches the boundary velocity in any direction. The parameters $\tau_{a}, \tau_{h}, \phi_{a}$ and $\phi_{h}$ depend, like the wavenumbers in Eqs. $(1,2,3)$, on physical constants and the frequency. The velocity equation (5) is a vector equation, which can be split for convenience into normal and tangential components:

$$
\begin{aligned}
& \vec{v}_{\text {boundary }, n}=\phi_{a} \frac{\partial p_{a}}{\partial n}+\phi_{h} \frac{\partial p_{h}}{\partial n}+\vec{v}_{v, n} \\
& \vec{v}_{\text {boundary }, t}=\phi_{a} \nabla_{t} p_{a}+\phi_{h} \nabla_{t} p_{h}+\vec{v}_{v, t}
\end{aligned}
$$

Note that Eqs. $(1,2,3)$ are of the same form as the lossless harmonic Helmholtz equation. The BEM implementation with losses starts by discretizing these three equations independently, using the same procedure as in the lossless BEM, that is, converting the Helmholtz equation into its integral form, ${ }^{16,17}$

$$
C(P) p(P)=\int_{S}\left[\frac{\partial G(Q)}{\partial n} p(Q)-\frac{\partial p(Q)}{\partial n} G(Q)\right] d S+p^{I}(P)
$$

where $p$ is the sound pressure, $G$ is the Green's function and $P$ and $Q$ are points in the domain and on the surface respectively. $C(P)$ is a geometrical constant and $p^{I}(P)$ is the incident pressure, if present. The boundary is then divided into surface elements and Eq. (8) is discretized as

$$
\mathbf{A p}-\mathbf{B} \frac{\partial \mathbf{p}}{\partial n}+\mathbf{p}^{I}=0
$$

Eq. (9) is defined over the boundary. Given a set of boundary conditions, it can be solved for obtaining the boundary pressure and normal particle velocity. The acoustic magnitudes in the domain are obtained from the surface solution by re-applying the discretized Helmholtz Integral Equation. 
The harmonic equations $(1,2,3)$ of the Kirchhoff decomposition can be discretized as

$$
\begin{gathered}
\mathbf{A}_{a} \mathbf{p}_{a}-\mathbf{B}_{a} \frac{\partial \mathbf{p}_{a}}{\partial n}+\mathbf{p}^{I}=0 \\
\mathbf{A}_{h} \mathbf{p}_{h}-\mathbf{B}_{h} \frac{\partial \mathbf{p}_{h}}{\partial n}=0 \\
\mathbf{A}_{v} \overrightarrow{\mathbf{v}}_{v}-\mathbf{B}_{v} \frac{\partial \overrightarrow{\mathbf{v}}_{v}}{\partial n}=\overrightarrow{0}, \text { with } \nabla \cdot \overrightarrow{\mathbf{v}}_{v}=0
\end{gathered}
$$

The development in Ref. [13] makes use of coordinate transformations between the node-based local reference system (normal and tangential vectors $n, t_{1}$ and $t_{2}$ ) and the global Cartesian reference $(x, y, z)$ to combine Eqs. $(10,11,12)$. The coupling conditions in Eqs. (4,5) and the null divergence of the viscous velocity in Eq. (12) are employed. The resulting system of equations for obtaining the acoustic component of the pressure on the boundary is

$$
\begin{gathered}
\\
{\left[\phi_{a} \mathbf{B}_{a}^{-1} \mathbf{A}_{a}-\phi_{h} \mathbf{B}_{h}^{-1} \mathbf{A}_{h} \frac{\tau_{a}}{\tau_{h}}+\right.} \\
\left.\left[\mathbf{N}_{11} \circ\left(\mathbf{B}_{v}^{-1} \mathbf{A}_{v}\right)\right]^{-1}\left(\phi_{a}-\frac{\tau_{a}}{\tau_{h}} \phi_{h}\right)\left(\left[\mathbf{N}_{12} \circ\left(\mathbf{B}_{v}^{-1} \mathbf{A}_{v}\right)\right] \frac{\partial}{\partial t_{1}}+\left[\mathbf{N}_{13} \circ\left(\mathbf{B}_{v}^{-1} \mathbf{A}_{v}\right)\right] \frac{\partial}{\partial t_{2}}+\Delta_{t}\right)\right] \mathbf{p}_{a}= \\
\overrightarrow{\mathbf{v}}_{\text {boundary }, n}+\left[\mathbf{N}_{11} \circ\left(\mathbf{B}_{v}^{-1} \mathbf{A}_{v}\right)\right]^{-1}\left[\left[\mathbf{N}_{12} \circ\left(\mathbf{B}_{v}^{-1} \mathbf{A}_{v}\right)\right]+\frac{\partial}{\partial t_{1}}\right] \overrightarrow{\mathbf{v}}_{\text {boundary }, t_{1}}+ \\
{\left[\mathbf{N}_{11} \circ\left(\mathbf{B}_{v}^{-1} \mathbf{A}_{v}\right)\right]^{-1}\left[\left[\mathbf{N}_{13} \circ\left(\mathbf{B}_{v}^{-1} \mathbf{A}_{v}\right)\right]+\frac{\partial}{\partial t_{2}}\right] \overrightarrow{\mathbf{v}}_{\text {boundary }, t_{2}}-\phi_{a} \mathbf{B}_{a}^{-1} \mathbf{p}^{I}}
\end{gathered}
$$

The "o" operator in Eq. (13) is the Hadamard matrix product and the constant matrices $\mathbf{N}_{11}, \mathbf{N}_{12}$ and $\mathbf{N}_{13}$ are obtained as

$$
\begin{gathered}
\mathbf{N}_{11}=\mathbf{n}_{x} \mathbf{n}_{x}^{T}+\mathbf{n}_{y} \mathbf{n}_{y}^{T}+\mathbf{n}_{z} \mathbf{n}_{z}^{T} \\
\mathbf{N}_{12}=\mathbf{n}_{x} \mathbf{t}_{1, x}^{T}+\mathbf{n}_{y} \mathbf{t}_{1, y}^{T}+\mathbf{n}_{z} \mathbf{t}_{1, z}^{T} \\
\mathbf{N}_{13}=\mathbf{n}_{x} \mathbf{t}_{2, x}^{T}+\mathbf{n}_{y} \mathbf{t}_{2, y}^{T}+\mathbf{n}_{z} \mathbf{t}_{2, z}^{T}
\end{gathered}
$$

where the right-hand sides contain products of the $(x, y, z)$ components of the node-based normal and tangential vectors $n, t_{1}$ and $t_{2}$.

Eq. (13) relates the prescribed normal and tangential velocities on the boundary $\left(\overrightarrow{\mathbf{v}}_{\text {boundary }, n}, \overrightarrow{\mathbf{v}}_{\text {boundary }, t_{1}}\right.$ and $\left.\overrightarrow{\mathbf{v}}_{\text {boundary }, t_{2}}\right)$ and the incident pressure $\mathbf{p}^{I}$ with the boundary pressures associated with the acoustic mode $\mathbf{p}_{a}$. After solving this system, it is possible to derive the remaining magnitudes on the boundary $\left(\mathbf{p}_{h}, \overrightarrow{\mathbf{v}}_{v}\right)$, and on the domain, as described in Ref. [13].

The $\frac{\partial}{\partial t_{1}}, \frac{\partial}{\partial t_{2}}$ and $\Delta_{t}$ operators in Eq. (13) are tangential derivatives and tangential laplacian respectively. They are implemented in Ref. [13] as matrices with a finite difference scheme based on Voronoi cells over non-regular meshes. ${ }^{18,19}$ This method implies that the finite difference step size is equivalent to the element dimensions of the boundary mesh. The 
implementation described in this section will be termed Finite Difference Derivative BEM (FDD-BEM).

\section{BEM implementation with losses using shape function derivatives}

It has been found that the finite difference scheme just described in the previous section can be detrimental for the performance of the BEM with viscous and thermal losses, as will be shown in the test case calculations further in this paper. In this section we propose a modified implementation that does not include finite differences. It is based on the same set of equations and coupling conditions in the previous section, but in this case the tangential derivatives are adapted from the analytical derivatives of the element shape functions.

In this paper, a direct collocation implementation of the BEM is used. ${ }^{20}$ The boundary surface is meshed with isoparametric quadratic triangular elements. Other elements could be used, providing they are second-order (quadratic) or higher. This ensures that the tangential derivative approximation is at least first-order (linear).

By using the chain rule derivation, the derivatives of the shape functions with respect to the local element coordinates can be converted into derivatives with respect to the global coordinates as ${ }^{21}$

$$
\left.\left\{\begin{array}{l}
\frac{\partial N_{i}}{\partial x} \\
\frac{\partial N_{i}}{\partial y} \\
\frac{\partial N_{i}}{\partial z}
\end{array}\right\}=\left[\begin{array}{lll}
\frac{\partial x}{\partial \xi_{1}} & \frac{\partial y}{\partial \xi_{1}} & \frac{\partial z}{\partial \xi_{1}} \\
\frac{\partial x}{\partial \xi_{2}} & \frac{\partial y}{\partial \xi_{2}} & \frac{\partial z}{\partial \xi_{2}} \\
\frac{\partial x}{\partial \xi_{3}} & \frac{\partial y}{\partial \xi_{3}} & \frac{\partial z}{\partial \xi_{3}}
\end{array}\right]^{-1} \quad \begin{array}{l}
\frac{\partial N_{i}}{\partial \xi_{1}} \\
\frac{\partial N_{i}}{\partial \xi_{2}} \\
\frac{\partial N_{i}}{\partial \xi_{3}}
\end{array}\right\}
$$

Where the inverted matrix in the right-hand side is the Jacobian and $N_{i}$ is a shape function. The derivatives $\left(\frac{\partial N_{i}}{\partial \xi_{1}}, \frac{\partial N_{i}}{\partial \xi_{2}}, \frac{\partial N_{i}}{\partial \xi_{3}}\right)$ are obtained analytically. Eq. (15) is written for three-dimensional elements, but it is possible to rewrite it for surface elements in threedimensional domains. Surface elements have only two independent local coordinates $\left(\xi_{1}, \xi_{2}\right)$, so the last row of the Jacobian matrix is obtained as the vector product of the first two rows. ${ }^{22}$ In our case, the tangential derivatives $\left(\frac{\partial}{\partial t_{1}}, \frac{\partial}{\partial t_{2}}\right)$ at the nodal positions are needed. The global $(x, y, z)$ coordinate system is therefore replaced by the node-related local coordinate system $\left(n, t_{1}, t_{2}\right)$ using a linear coordinate change. This procedure is repeated for all shape functions on every node. We calculate the two tangential derivatives of the viscous velocity vector as

$$
\begin{gathered}
\frac{\partial \mathbf{v}_{v, x}}{\partial t_{1}}=\sum_{i}\left(\frac{\partial N_{i}}{\partial t_{1}} v_{v, x, i}\right), \frac{\partial \mathbf{v}_{v, y}}{\partial t_{1}}=\sum_{i}\left(\frac{\partial N_{i}}{\partial t_{1}} v_{v, y, i}\right), \frac{\partial \mathbf{v}_{v, z}}{\partial t_{1}}=\sum_{i}\left(\frac{\partial N_{i}}{\partial t_{1}} v_{v, z, i}\right), \\
\frac{\partial \mathbf{v}_{v, x}}{\partial t_{2}}=\sum_{i}\left(\frac{\partial N_{i}}{\partial t_{2}} v_{v, x, i}\right), \frac{\partial \mathbf{v}_{v, y}}{\partial t_{2}}=\sum_{i}\left(\frac{\partial N_{i}}{\partial t_{2}} v_{v, y, i}\right), \frac{\partial \mathbf{v}_{v, z}}{\partial t_{2}}=\sum_{i}\left(\frac{\partial N_{i}}{\partial t_{2}} v_{v, z, i}\right)
\end{gathered}
$$

where $v_{v, x}, v_{v, y}$ and $v_{v, z}$ are the Cartesian components of the viscous velocity vector. Their tangential derivatives are expressed as a function of the nodal values $v_{v, x, i}, v_{v, y, i}$ and $v_{v, z, i}$. Note that, if the elements are continuous, nodes can be shared by several elements. In 
this case, the contributions to the tangential derivatives from all elements the node belongs to are averaged. The tangential derivatives of the acoustic pressure are obtained in the same way,

$$
\frac{\partial p_{a}}{\partial t_{1}}=\sum_{i}\left(\frac{\partial N_{i}}{\partial t_{1}} p_{a, i}\right), \frac{\partial p_{a}}{\partial t_{2}}=\sum_{i}\left(\frac{\partial N_{i}}{\partial t_{2}} p_{a, i}\right)
$$

The viscous velocity is a rotational vector field; its divergence is zero $\left(\frac{\partial \mathbf{v}_{v, n}}{\partial n}+\frac{\partial \mathbf{v}_{v, t_{1}}}{\partial t_{1}}+\right.$ $\left.\frac{\partial \mathbf{v} v, t_{2}}{\partial t_{2}}=0\right)$. The three terms of this divergence can be expressed as

$$
\begin{gathered}
\frac{\partial \mathbf{v}_{v, n}}{\partial n}=\mathbf{n}_{x} \circ\left(\mathbf{B}_{v}^{-1} \mathbf{A}_{v} \mathbf{v}_{v, x}\right)+\mathbf{n}_{y} \circ\left(\mathbf{B}_{v}^{-1} \mathbf{A}_{v} \mathbf{v}_{v, y}\right)+\mathbf{n}_{z} \circ\left(\mathbf{B}_{v}^{-1} \mathbf{A}_{v} \mathbf{v}_{v, z}\right) \\
\frac{\partial \mathbf{v}_{v, t_{1}}}{\partial t_{1}}=\mathbf{t}_{1, x} \circ \sum_{i}\left(\frac{\partial N_{i}}{\partial t_{1}} v_{v, x, i}\right)+\mathbf{t}_{1, y} \circ \sum_{i}\left(\frac{\partial N_{i}}{\partial t_{1}} v_{v, y, i}\right)+\mathbf{t}_{1, z} \circ \sum_{i}\left(\frac{\partial N_{i}}{\partial t_{1}} v_{v, z, i}\right) \\
\frac{\partial \mathbf{v}_{v, t_{2}}}{\partial t_{2}}=\mathbf{t}_{2, x} \circ \sum_{i}\left(\frac{\partial N_{i}}{\partial t_{2}} v_{v, x, i}\right)+\mathbf{t}_{2, y} \circ \sum_{i}\left(\frac{\partial N_{i}}{\partial t_{2}} v_{v, y, i}\right)+\mathbf{t}_{2, z} \circ \sum_{i}\left(\frac{\partial N_{i}}{\partial t_{2}} v_{v, z, i}\right)
\end{gathered}
$$

Eq. (18) is obtained by taking the normal derivative of the coordinate change $\mathbf{v}_{v, n}=$ $\mathbf{n}_{x} \circ \mathbf{v}_{v, x}+\mathbf{n}_{y} \circ \mathbf{v}_{v, y}+\mathbf{n}_{z} \circ \mathbf{v}_{v, z}$ and replacing the three Cartesian components of Eq. (12). Eqs. $(19,20)$ are the result of replacing the relations of Eq. (16) in the tangential derivatives of the remaining coordinate change equations $\mathbf{v}_{v, t_{1}}=\mathbf{t}_{1, x} \circ \mathbf{v}_{v, x}+\mathbf{t}_{1, y} \circ \mathbf{v}_{v, y}+\mathbf{t}_{1, z} \circ \mathbf{v}_{v, z}$ and $\mathbf{v}_{v, t_{2}}=\mathbf{t}_{2, x} \circ \mathbf{v}_{v, x}+\mathbf{t}_{2, y} \circ \mathbf{v}_{v, y}+\mathbf{t}_{2, z} \circ \mathbf{v}_{v, z}$.

Using the properties of the Hadamard operator "०", Eqs. $(18,19,20)$ can be rearranged as

$$
\begin{gathered}
\frac{\partial \mathbf{v}_{v, n}}{\partial n}=\left[\left(\mathbf{n}_{x} \mathbf{I}\right) \circ\left(\mathbf{B}_{v}^{-1} \mathbf{A}_{v}\right)\right] \mathbf{v}_{v, x}+\left[\left(\mathbf{n}_{y} \mathbf{I}\right) \circ\left(\mathbf{B}_{v}^{-1} \mathbf{A}_{v}\right)\right] \mathbf{v}_{v, y}+\left[\left(\mathbf{n}_{z} \mathbf{I}\right) \circ\left(\mathbf{B}_{v}^{-1} \mathbf{A}_{v}\right)\right] \mathbf{v}_{v, z} \\
\frac{\partial \mathbf{v}_{v, t_{1}}}{\partial t_{1}}=\left[\left(\mathbf{t}_{1, x} \mathbf{I}\right) \circ \mathbf{D}_{t_{1}}\right] \mathbf{v}_{v, x}+\left[\left(\mathbf{t}_{1, y} \mathbf{I}\right) \circ \mathbf{D}_{t_{1}}\right] \mathbf{v}_{v, y}+\left[\left(\mathbf{t}_{1, z} \mathbf{I}\right) \circ \mathbf{D}_{t_{1}}\right] \mathbf{v}_{v, z} \\
\frac{\partial \mathbf{v}_{v, t_{2}}}{\partial t_{2}}=\left[\left(\mathbf{t}_{2, x} \mathbf{I}\right) \circ \mathbf{D}_{t_{2}}\right] \mathbf{v}_{v, x}+\left[\left(\mathbf{t}_{2, y} \mathbf{I}\right) \circ \mathbf{D}_{t_{2}}\right] \mathbf{v}_{v, y}+\left[\left(\mathbf{t}_{2, z} \mathbf{I}\right) \circ \mathbf{D}_{t_{2}}\right] \mathbf{v}_{v, z}
\end{gathered}
$$

where $\mathbf{D}_{t_{1}}$ and $\mathbf{D}_{t_{2}}$ are matrices implementing the tangential derivatives and $\mathbf{I}$ is the identity matrix. We may also impose the no-slip condition Eq. (7) by using Eq. (17). After some manipulation it becomes

$$
\begin{aligned}
& \overrightarrow{\mathbf{v}}_{\text {boundary }, t_{1}}=\left[\left(\phi_{a}-\frac{\tau_{a}}{\tau_{h}} \phi_{h}\right) \mathbf{D}_{t_{1}}\right] \mathbf{p}_{a}+\left(\mathbf{t}_{1, x} \mathbf{I}\right) \mathbf{v}_{v, x}+\left(\mathbf{t}_{1, y} \mathbf{I}\right) \mathbf{v}_{v, y}+\left(\mathbf{t}_{1, z} \mathbf{I}\right) \mathbf{v}_{v, z} \\
& \overrightarrow{\mathbf{v}}_{\text {boundary }, t_{2}}=\left[\left(\phi_{a}-\frac{\tau_{a}}{\tau_{h}} \phi_{h}\right) \mathbf{D}_{t_{2}}\right] \mathbf{p}_{a}+\left(\mathbf{t}_{2, x} \mathbf{I}\right) \mathbf{v}_{v, x}+\left(\mathbf{t}_{2, y} \mathbf{I}\right) \mathbf{v}_{v, y}+\left(\mathbf{t}_{2, z} \mathbf{I}\right) \mathbf{v}_{v, z}
\end{aligned}
$$


Finally, the normal velocity coupling condition Eq. (6) can be arranged as $\overrightarrow{\mathbf{v}}_{\text {boundary }, n}=\left[\phi_{a} \mathbf{B}_{a}^{-1} \mathbf{A}_{a}-\phi_{h} \mathbf{B}_{h}^{-1} \mathbf{A}_{h} \frac{\tau_{a}}{\tau_{h}}\right] \mathbf{p}_{a}+\left(\mathbf{n}_{x} \mathbf{I}\right) \mathbf{v}_{v, x}+\left(\mathbf{n}_{y} \mathbf{I}\right) \mathbf{v}_{v, y}+\left(\mathbf{n}_{z} \mathbf{I}\right) \mathbf{v}_{v, z}+\phi_{a} \mathbf{B}_{a}^{-1} \mathbf{p}^{I}$

The preceding equations are combined into a system of equations of the form

$$
\left[\begin{array}{c}
\text { Normal velocity coupling, Eq. (26) } \\
\text { Null divergence of } \overrightarrow{\mathbf{v}}_{v}, \text { Eqs. }(21,22,23) \\
\text { No-slip condition } t_{1} \text {, Eq. (24) } \\
\text { No-slip condition } t_{2} \text {, Eq. (25) }
\end{array}\right]\left\{\begin{array}{c}
\mathbf{p}_{a} \\
\mathbf{v}_{v, x} \\
\mathbf{v}_{v, y} \\
\mathbf{v}_{v, z}
\end{array}\right\}=\left\{\begin{array}{c}
\overrightarrow{\mathbf{v}}_{\text {boundary }, n}-\phi_{a} \mathbf{B}_{a}^{-1} \mathbf{p}^{I} \\
0 \\
\overrightarrow{\mathbf{v}}_{\text {boundary }_{1}} \\
\overrightarrow{\mathbf{v}}_{\text {boundary }, t_{2}}
\end{array}\right\}
$$

Note that the system in Eq. (27) has four sets of unknowns, $\mathbf{p}_{a}, \mathbf{v}_{v, x}, \mathbf{v}_{v, y}$ and $\mathbf{v}_{v, z}$, while the original system in Eq. (13) is solved for $\mathbf{p}_{a}$ alone. This means a coefficient matrix that is $4 \times 4$ times larger, but avoids the need of a second order tangential derivative. The system is solved, as for FDD-BEM, using the MATLAB backslash operator. The expanded coefficient matrix is not fully populated: in the sphere test case only about $1 / 3$ of the matrix coefficients in Eq. (27) are non-zero. The solving could be made more efficient by making use of sparsity, e.g. by using dedicated solvers.

From the solution of Eq. (27) it is possible to obtain all other magnitudes on the boundary and the domain using the procedure described in Ref. [13]. This new version of the BEM with losses will be called Shape Function Derivative BEM (SFD-BEM).

In order to evaluate independently the effect of using shape function derivatives and the expanded system of equations of Eq. (27), a combined version of the two methods is also employed. It is named FDD2-BEM, and it uses the finite difference tangential derivatives from section 2 into Eq. (27).

\section{Oscillating sphere test case}

The performance of the SFD-BEM against the FDD-BEM is studied using a test case containing losses: a sphere with a radius of $1 \mathrm{~m}$ that radiates sound by oscillating as a whole with a velocity of $10^{-2} \mathrm{~m} / \mathrm{s}$ along the z-axis. Only the results at the boundary are calculated; domain results are possible, but less suitable for the purpose of this study. This test case has an analytical solution and has been previously used for testing the axisymmetrical version of the BEM with losses. ${ }^{23,12}$ Note that the analytical solution does not consider thermal losses. This is not however a problem since the main difficulties of all formulations with losses lie in the viscous mode, which is largely dominant in this test case.

The boundary mesh is generated using the software package Gmsh. ${ }^{24}$ It contains 2406 nodes and 1202 quadratic 6-node triangular elements and is shown in Fig. 1. The Gmsh initial surface element size is specified as $1 / 7$ times the sphere radius.

The oscillating sphere is centered on the origin, and generates a viscous mode velocity on its surface, which is larger and tangential to the boundary at its equator $(z=0)$. The tangential component of this viscous velocity must cancel the acoustic and thermal tangential velocities so as to fulfill the no-slip condition. The normal component of the 


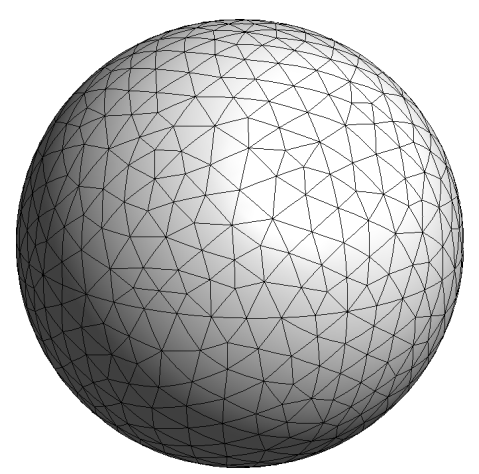

Fig. 1. BEM mesh of the sphere employed in the test case. It contains 2406 nodes and 1202 elements.

viscous velocity is much smaller, and larger the closer to the poles $(z= \pm 1)$. Four frequencies have been calculated: $200 \mathrm{~Hz}, 500 \mathrm{~Hz}, 800 \mathrm{~Hz}$ and $1000 \mathrm{~Hz}$. For these frequencies and the mesh in Fig. 1, the approximate numbers of nodes per wavelength are 22, 8.8, 5.4 and 4.4 respectively, so that the last two are below the 6 nodes per wavelength rule of thumb. The frequencies are chosen away from internal eigenfrequencies of the sphere so as to avoid the non-uniqueness problem of the BEM. ${ }^{22}$

Fig. 2 shows results on the surface of the acoustic mode pressure and the normal and tangential components of the viscous mode velocity at $1 \mathrm{kHz}$. The FDD-BEM fails to calculate the relatively small normal component of the viscous velocity, as can be seen in the mid-left plot. The combined version FDD2-BEM using finite differences in the expanded system of equations in Eq. (27), brings some improvement, as shown in the central plot. Only the SFD-BEM, in the left column graphs, calculates all three magnitudes satisfactorily. Comparison of FDD-BEM and FDD2-BEM allows estimating the effect of expanding the system of equations from Eq. (13) to Eq. (27), while the comparison of FDD2-BEM and SFD-BEM shows the effect of using shape function tangential derivatives over finite differences. Simlar results are obtained at the remaining calculated frequencies, leading to the same conclusions. The difficulty in the calculation of the normal component of the viscous velocity may be a consequence of its relatively small amplitude, making it more seriously affected by machine errors.

Fig. 3 examines the relative error for the three formulations (FDD-BEM, SFD-BEM and the combined FDD2-BEM) at four different frequencies. In order to avoid difficulties when the solution is close to zero, the error measure is made relative to the maximum absolute value of the analytical solution over the geometry as

$$
\text { Error }=\sum_{i} \frac{\mid \text { Analytical }_{i}-\text { Calculated }_{i} \mid}{\mid \max (\text { Analytical }) \mid}
$$

The ideal expected behavior of the errors would be a steady increase as the frequency grows and the mesh is kept constant. 

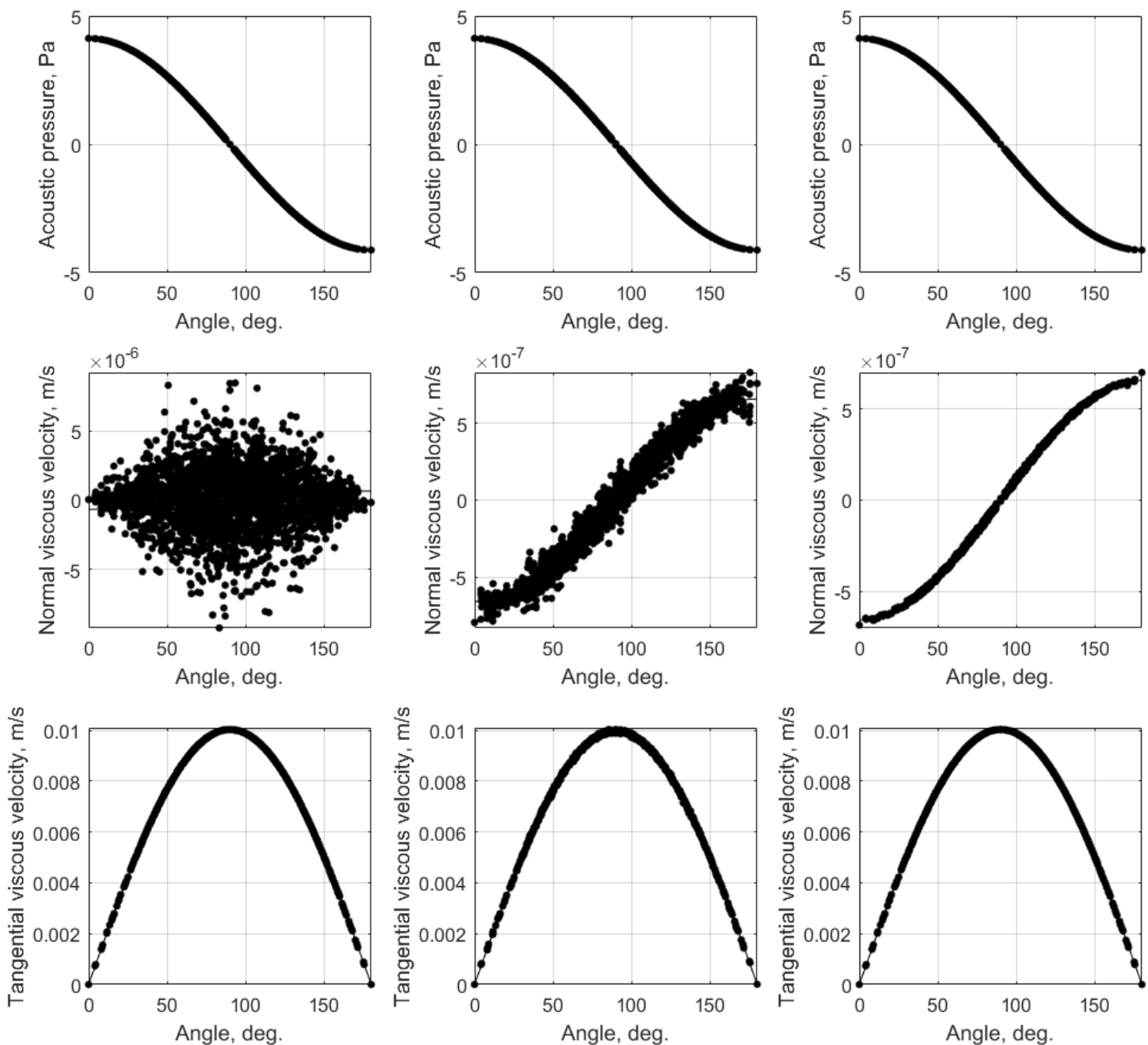

Fig. 2. Solution on the surface over an arc from pole to pole (from $z=1$ to $z=-1$ ) of an oscillating sphere, at $1 \mathrm{kHz}$. The plots on the left column are calculated using the existing FDD-BEM, the plots in the central column use the FDD2-BEM (finite differences combined with Eq. (27)), and the plots on the right column come from the new SFD-BEM. The values are all real parts of the indicated magnitudes. The underlying solid line is the analytical solution, and the dots are BEM results on the mesh nodes. Note the different scales on the normal viscous velocity plots.

\section{Microphone test case}

In many cases, the SFD-BEM performance is similar to the original FDD-BEM formulation, for example a metamaterial model. ${ }^{6}$ However, the SFD-BEM shows its benefits in cases where the calculation is affected by several sources of instability. In this section we use a three-dimensional BEM model of a condenser microphone as a test case. Such a model has to cope with: i) a very narrow gap behind the membrane with a thickness that is comparable 

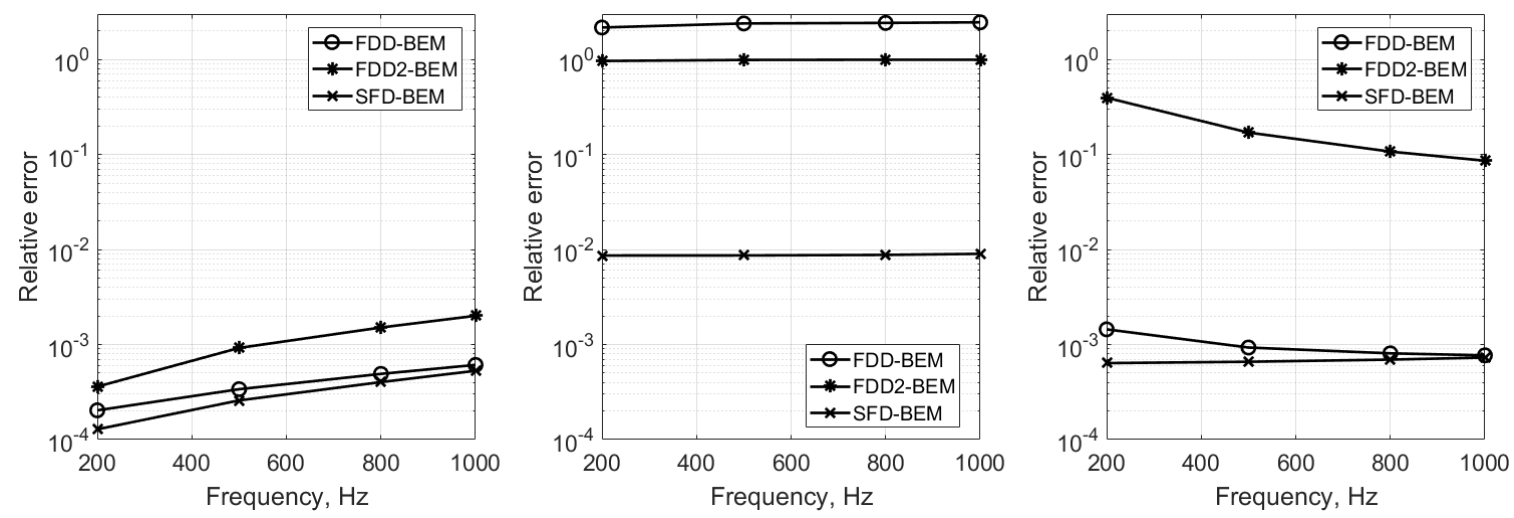

Fig. 3. Relative error at different frequencies of the BEM boundary solution for an oscillating sphere of $1 \mathrm{~m}$ radius with the mesh in Fig. 1. The circles correspond to the FDD-BEM, the crosses are obtained with the SFD-BEM and the asterisks are the combined formulation FDD2-BEM. The left-side plot is the acoustic pressure error, the central plot is the normal viscous velocity error, and the right-side plot is the tangential viscous velocity error.

to the thicknesses of the viscous and thermal boundary layers at the membrane resonance frequency, ii) the coupling of a FEM model of the membrane and a BEM model with losses of the interior, iii) the intrinsic instability of the BEM for closed cavities. ${ }^{25}$
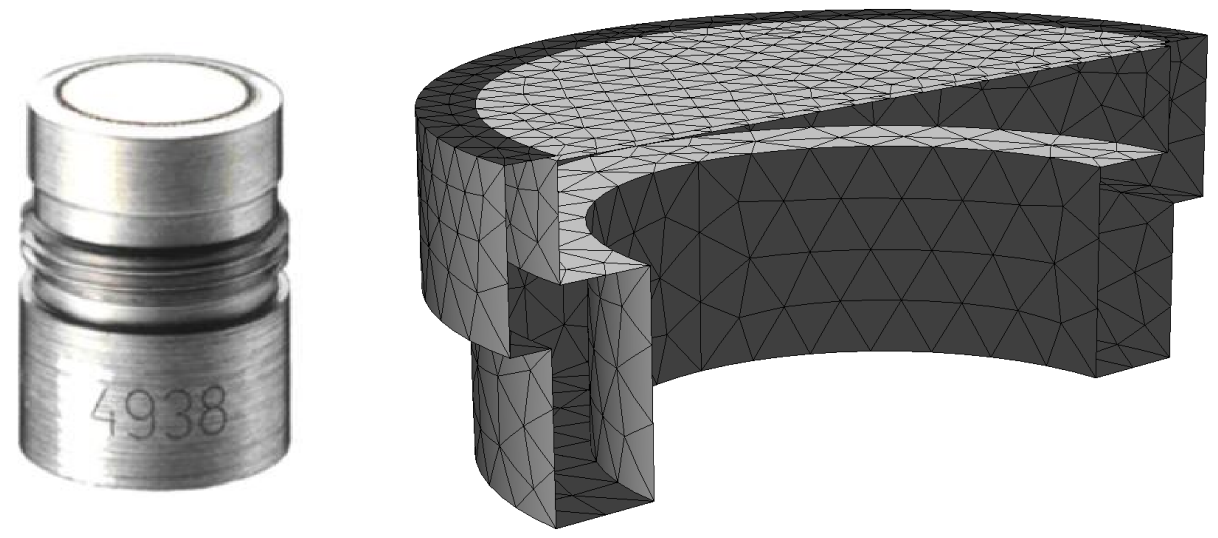

Fig. 4. Photograph (left) and BEM mesh (right) of a Brüel\&Kjær microphone type 4938, as in Ref. [26]. The mesh has 6322 nodes and 3160 quadratic triangular elements. The membrane is modeled with a twodimensional FEM and coupled to the interior. The mesh has been cut in half to show the interior with the narrow gap, the back plate electrode and the ring-shaped back cavity.

We revisit here the model of a measurement condenser microphone Brüel\&Kjær type 4938 that was used as an example in Ref. [26]. This is a $\frac{1}{4}$ inch pressure-field microphone with a gap thickness of $20 \mu \mathrm{m}$ and a resonance frequency of the membrane of $60 \mathrm{kHz}$. The microphone and the corresponding BEM mesh are represented in Fig. 4. The interior of the microphone is modeled using BEM with losses, and coupled to a membrane, which is 


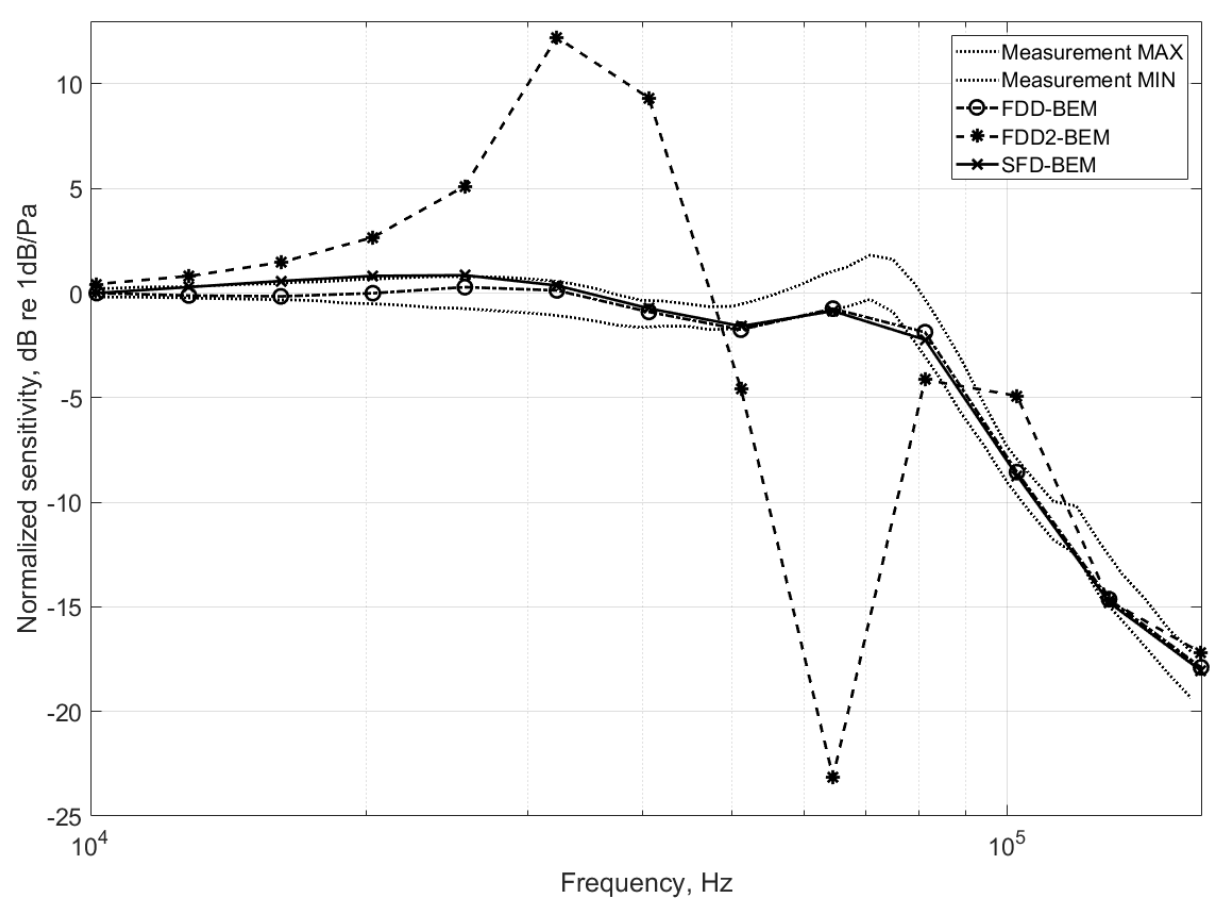

Fig. 5. Normalized sensitivity response with actuator excitation of a Brüel\&Kjær microphone type 4938. The dotted lines represent the upper and lower bounds of of the measured sensitivities of 183 microphone units. The circles correspond to the FDD-BEM, the crosses are obtained with the SFD-BEM and the asterisks are the combined formulation FDD2-BEM.

modeled with a two-dimensional FEM, also written for this purpose. The excitation is made to resemble an electrostatic actuator measurement of the microphone sensitivity response by setting a uniform sound pressure over the membrane. In Ref. [26], a node in the back cavity (vent node) was given a finite impedance to stabilize the calculation. We have removed the vent node and recalculated this microphone using SFD-BEM, FDD-BEM and FDD2-BEM in the frequency range around the membrane resonance. The result is shown in Fig. 5. Note that it is not possible to match the model with particular measurement results, given the uncertainties in the model input parameters.

\section{Discussion}

The three formulations analyzed in this paper, the existing FDD-BEM, the new SFD-BEM and the combined FDD2-BEM are studied through two test cases, an oscillating sphere where an analytical solution exists and a measurement condenser microphone.

\subsection{Oscillating sphere test case}

In view of the results in Figs. 2 and 3, we can make the following observations:

(1) The SFD-BEM consistently produces smaller errors than the other two formulations 
for the three magnitudes examined, as shown in Fig. 3.

(2) As expected from observation of Fig. 2, the errors in the calculation of the normal component of the viscous velocity are greater in the case of FDD-BEM, followed by FDD2-BEM and SFD-BEM.

(3) Again in Fig. 3 (right-side plot), the FDD-BEM and FDD2-BEM have errors of the tangential component of the viscous velocity that actually decrease as frequency increases, the reason probably being that finite differences have larger numerical errors as the wavelength grows and the difference is closer to the machine precision, counterbalancing the error reduction due to a better discretization.

(4) In the calculation of the tangential component of the viscous velocity and the pressure, FDD-BEM shows smaller errors than FDD2-BEM, indicating that the use of the expanded system of equations in Eq. (27) comes with some cost.

(5) The SFD-BEM has a larger error (yet below 1\%) for the normal component of the viscous velocity than for the other two magnitudes. As pointed out in section 4, its relatively lower amplitude may be an explanation, together with the fact that the shape function derivatives follow a linear approximation rather than quadratic.

The error behavior of the three shown magnitudes is different as a result of the involved calculation process. Only the acoustic pressure, which is the first obtained result in all formulations, has an error that grows with the frequency. All in all, the results shown in Figs. 2 and 3 indicate that the expansion of the system of equations Eq. (27) and the new strategy using shape functions for the tangential derivatives are advantageous over the other strategies when combined into the SFD-BEM.

\subsection{Condenser microphone test case}

Condenser microphones rely on the viscous and thermal losses in the gap between membrane and back electrode for the correct damping of the membrane resonance: a model with losses is necessary. As mentioned in section 5, the BEM, unlike FEM, becomes unstable for closed interior domains, particularly at mid-low frequencies where the interior pressure tends to be uniform. ${ }^{25}$ This problem affects the BEM with or without losses. We have experienced this issue as a source of instability in all closed microphone models, and we have dealt with it in previous publications by adding a finite impedance to one of the nodes in the back cavity, resembling the vent all condenser measurement microphones have. We are working on more fundamental solutions to this flaw of the BEM, since the vent node just forces the pressure to vary in the back cavity without addressing the intrinsic instability of the BEM.

The model should be able to run without a vent node, but the fact is that its removal makes the BEM calculation more prone to instability, as the results presented here confirm. The formulations that are based on finite differences have difficulties to overcome the instability, and this manifests as a system of equations that is close to being singular. In the case of the FDD-BEM, the MATLAB solver is just able to cope with the solution, however issuing warnings on the unreliability of the solution. The expanded system, Eq. (27), in the FDD2-BEM adds some extra difficulty that makes the solver fail; this is compatible with 
the observation made in item (4) in subsection 6.1, about the oscillating sphere results. The solver has no difficulty with the new SFD-BEM, which shows to be more stable and succeeds in providing a solution despite using the expanded system of equations in Eq. (27).

\section{Conclusions and future work}

A modified implementation of the Boundary Element Method for acoustic waves with viscous and thermal losses is presented in this paper. The technique based in two-dimensional finite differences employed in the previous version ${ }^{13}$ of BEM with losses is replaced by analytical derivatives of the element shape functions, thus simplifying the implementation and making it less prone to errors. This is demonstrated through two test cases: an oscillating sphere, which shows a clear improvement for the same mesh density, and a condenser microphone model, where the intrinsic instability of the setup threatens the solution when finite difference tangential derivatives are used. The new shape function tangential derivatives perform well in both test cases.

The benefits of the technique come with some drawbacks. The coefficient matrix of the system of equations is $4 \times 4$ times larger, since it must be expanded from one to four sets of unknowns. However, obtaining the six coefficient matrices for the three visco-thermal modes in Eqs. (10-12) takes most of the calculation effort and this is the same for both formulations. Another issue is the need for the order of the elements to be sufficient for a good representation of the tangential derivatives, which are one order lower than the shape functions. The quadratic elements employed lead to a linear representation of the derived magnitudes.

The formulation presented here is defined in three dimensions. BEM formulations with losses, based on finite differences, also exist for two-dimensional and axisymmetric problems. ${ }^{27,12}$ The shape function derivative technique has not been thoroughly tested in these cases. However, considering that the one-dimensional finite difference scheme employed in two-dimensional and axisymmetric implementations is much more straightforward and precise in these cases, less benefit is expected.

A possible future refinement of the technique is the use of discontinuous elements, where element nodes are not shared. ${ }^{28,29,30}$ The shape function derivative formulation takes an average of the contributions to normal vectors and tangential derivatives from all the elements a node belongs to; this would be avoided by using discontinuous elements, and may lead to even better performance. Another possible improvement is the use of elements of higher orders than quadratic, which would improve the representation of the tangential derivative.

\section{References}

1. J.W.S. Rayleigh, The theory of sound (Dover, 1945, reprinted from $2^{\text {nd }}$ ed., 1894).

2. A. D. Pierce, Acoustics. An introduction to its physical principles and applications (Ch. 10) (McGraw Hill, New York, 1981).

3. P. M. Morse and K. U. Ingard, Theoretical Acoustics (McGraw-Hill, Princeton, 1968). 
4. V. Cutanda Henríquez, P. M. Juhl, Modelling measurement microphones using BEM with viscothermal losses. Joint Baltic-Nordic Acoustics Meeting, 18-20 June 2012, Odense, Denmark.

5. V. Cutanda Henríquez, S. Barrera-Figueroa, A. Torras Rosell, P. M. Juhl, Study of the acoustical properties of a condenser microphone under an obliquely incident plane wave using a fully coupled three-dimensional numerical model. Internoise 2015, 9-12 Aug. 2015, San Francisco (USA).

6. V. Cutanda Henríquez, P. R. Andersen, J. S. Jensen, P. M. Juhl and J. Sánchez-Dehesa, A numerical model of an acoustic metamaterial using the Boundary Element Method including viscous and thermal losses, Journal of Computational Acoustics, vol 25, no. 4 (2016).

7. R. Bossart, N. Joly and M. Bruneau. Methodes de modelisation numerique des champs acoustiques en fluide thermovisqueux. Actes du $6^{e}$ Congrès Franais d'Acoustique, Lille, France (2002), pp. 411-414.

8. M. Malinen, M. Lyly, P. Råback, A. Kärkkäinen and L. Kärkkäinen, A finite element method for the modeling of thermo-viscous effects in acoustics. Proc. $4^{\text {th }}$ European Congress Computational Methods in Applied Sciences and Engineering ECCOMAS, Jyväskylä, Finland (2004).

9. N. Joly, Coupled equations for particle velocity and temperature variation as the fundamental formulation of linear acoustics in thermo-viscous fluids at rest. Acta Acust. Acust. 92, 202209, (2006).

10. R. Kampinga, Performance of several viscothermal acoustic finite elements. Acta Acust. Acust. 96, 115124, (2010).

11. COMSOL Multiphysics Reference Manual, version 5.2 (2015).

12. V. Cutanda Henríquez and P. M. Juhl, An axisymmetric boundary element formulation of sound wave propagation in fluids including viscous and thermal losses. J. Acoust. Soc. Am., 134(5), 3409-3418, (2013).

13. V. Cutanda Henríquez and P. M. Juhl, Implementation of an Acoustic 3D BEM with ViscoThermal Losses, Internoise 2013, 15-18 Sept. 2013, Innsbruck, Austria.

14. M. Bruneau, Ph. Herzog, J. Kergomard and J. D. Polack, General formulation of the dispersion equation in bounded visco-thermal fluid, and application to some simple geometries, Wave Motion 11, 441-451 (1989).

15. P. R. Andersen, V. Cutanda Henríquez, N. Aage and S. Marburg, An Acoustic Hypersingular Boundary Element Formulation Including Viscous and Thermal Losses, $13^{\text {th }}$ International Conference on Theoretical and Computational Acoustics, 30 ${ }^{\text {th }}$ Jul. - $3^{\text {rd }}$ Aug. 2017, Vienna, Austria.

16. T. W. Wu (Ed.), Boundary Element Acoustics (WIT Press, 2000)

17. P. M. Juhl, The boundary Element Method for Sound Field Calculations, Ph.D. thesis, Report No. 55, Technical University of Denmark, 1993.

18. N. Sukumar, J. E. Bolander, Numerical computation of discrete differential operators on nonuniform grids, Comp. Model. in Eng. \& Sci., Vol. 4 Issue 6, 691-705 (2003).

19. C. Grossmann and H. G. Roos, Numerical treatment of partial differential equations (Springer, Berlin, 2007), Section 2.5.

20. V. Cutanda Henríquez and P. M. Juhl, OpenBEM - An open source Boundary Element Method software in Acoustics, Internoise 2010, 13-16 Jun. 2010, Lisbon, Portugal.

21. O. C. Zienkiewic and R. L. Taylor, The finite element method (Butterworth Heinemann, 2000). Section 9.5.

22. N. Atalla and F. Sgard, Finite element and boundary methods in structural acoustics and vibration (CRC Press, 2015). Sections 5.2.3.2.2 and 7.10.

23. S. Temkin, Elements of Acoustics (Wiley, New York, 1981), Sec. 6.9.

24. C. Geuzaine and J.-F. Remacle. Gmsh: a three-dimensional finite element mesh generator with built-in pre- and post-processing facilities. International Journal for Numerical Methods in Engineering 79(11), pp. 1309-1331, 2009.

25. S. Sorokin and S. T. Christensen, Low-frequency breakdown of boundary element formulation 
for closed cavities in excitation conditions with a 'breathing'-type component. Communications in Numerical Methods in Engineering 16, 325-334 (2000).

26. P. M. Juhl and V. Cutanda Henríquez, Verification of an Acoustic 3D BEM with Visco-Thermal Losses, Internoise 2013, 15-18 Sept. 2013, Innsbruck, Austria.

27. P. R. Andersen, V. Cutanda Henríquez, N. Aage and S.Marburg, Numerical Acoustic Models Including Viscous and Thermal losses: Review of Existing and New Methods, Proceedings of DAGA 2017. Deutsche Gesellschaft fr Akustik e.V (2017).

28. S. Marburg and S. Schneider, Influence of element types on numerical error for acoustic boundary elements. Journal of Computational Acoustics, Vol. 11, No. 3. (2003), pp. 363-386.

29. L. Chen, H. Chen, C. Zheng and S. Marburg, Structural-acoustic sensitivity analysis of radiated sound power using a finite element/ discontinuous fast multipole boundary element scheme. International Journal for Numerical Methods in Fluids, 82(12), pp. 858-878 (2016).

30. L. Chen, S. Marburg, H. Chen, H. Zhang and H. Gao, An Adjoint Operator Approach for Sensitivity Analysis of Radiated Sound Power in Fully Coupled Structural-Acoustic Systems. Journal of Computational Acoustics, Vol. 25, No. 1. (2017), 1750003. 